Paper

\title{
Information flow in learning a coin-tossing
}

\section{game}

\author{
Yuzuru Sato ${ }^{1,2 a)}$ and Nihat Ay ${ }^{3,4,5 b)}$ \\ ${ }^{1}$ RIES / Department of Mathematics, Hokkaido Univeristy \\ Kita 20 Nishi 10, Kita-ku, Sapporo 001-0020, Japan \\ ${ }^{2}$ London Mathematical Laboratory, 14 Buckingham Street, London, WC2N \\ $6 D F, U K$ \\ ${ }^{3}$ Max Planck Institute for Mathematics in the Sciences \\ Inselstrasse 22, D-04103 Leipzig, Germany \\ 4 Santa Fe Institute \\ 1399 Hyde Park Road, NM 87501, USA \\ ${ }^{5}$ Faculty of Mathematics and Computer Science, University of Leipzig \\ PF 100920, 04009 Leipzig, Germany \\ a)ysato@math.sci.hokudai.ac.jp \\ b) nay@mis.mpg.de
}

Received June 25, 2015; Revised November 10, 2015; Published April 1, 2016

\begin{abstract}
Information flow in adaptively interacting stochastic processes is studied. We give an extended form of game dynamics for interacting Markovian processes and compute a measure of causal information flow, which is different from the transfer entropy. In the game theoretic situation, causal information flow can show oscillatory behavior through reward-maximizing adaptation of two players. The adaptive dynamics for the coin-tossing game is exemplified and the causal information flow therein is investigated.
\end{abstract}

Key Words: game dynamics, information theory, dynamical systems

\section{Introduction}

When studying the interaction and evolution of many stochastic processes that are endowed with the ability to adapt to their environment, a natural question arises: how does information flow though the system and, moreover, how can we measure the information flow? From the viewpoint of networks of stochastic elements, flow of information in the network has been studied $[2,16]$. In general, mutual information is not a representative measure of information flow in adaptive dynamics as its causal structure forms a complex network, making the concept of information flow unclear. To address this problem, we give an example of game dynamics for interacting Markovian processes [3-6] and investigate information flow quantitatively. Suppose that $N$ stochastic processes $X_{1}, \ldots, X_{N}$ are interacting with each other. At each time step $\tau$, the unit $n$ sends a symbol $s_{n} \in\{0,1\}$ to the other 
units and receives at most $N-1$ symbols from the other units. We denote the global system state as $s=s_{1} \cdots s_{N}$. The next symbol sent by the unit $n, s_{n}^{\prime}$, is dependent on the symbol received from the previous global state, $s$. Local transition probabilities for $n$-th unit are described as

$$
x_{s_{n^{\prime} \mid s}}^{(n)}=P\left(X_{n}(\tau+1)=s_{n}{ }^{\prime} \mid X_{1}(\tau)=s_{1}, \ldots, X_{N}(\tau)=s_{N}\right),
$$

where $n=1, \ldots, N$ and $x_{0 \mid s}^{(n)}+x_{1 \mid s}^{(n)}=1$. The transition probabilities $\left(x_{0 \mid s}^{(n)}, x_{1 \mid s}^{(n)}\right)$ is an element of $N$ simplices denoted by $\Delta_{s}^{(n)}$.

We introduce a local adaptation process to change transition probabilities $x_{s_{n}{ }^{\prime} \mid s}^{(n)}$, assuming that adaptation is very slow compared with relaxation time of the global Markovian process. After the system reaches a stationary state, each unit independently changes its stochastic structure by changing its transition probabilities. Assuming strong connectivity of the global Markovian kernel, we study dynamics with strictly positive transition probabilities. This assumption corresponds to persistency of dynamics of transition probabilities $x_{s_{n}^{\prime} \mid s}^{(n)}$ in the state space. Time evolution of $x_{s_{n}^{\prime} \mid s}^{(n)}$ is driven by simple stochastic learning through interaction: reinforcements for transition probabilities of the unit $n$ to send 0 and 1 in the previous global state $s$ are given by the constants $a_{0 \mid s}^{(n)}$ and $a_{1 \mid s}^{(n)}$. The conditional expectation reinforcements $R_{s_{n}{ }^{\prime} \mid s}^{(n)}$ to choose each symbols $s_{n}{ }^{\prime}$ given the previous state $s$ are calculated with $a_{s_{n^{\prime} \mid} \mid s}^{(n)}, x_{s_{n^{\prime} \mid s}}^{(n)}$, and the unique stationary distribution. For $X_{n}$, we give adaptive dynamics for probabilities of $s_{n}^{\prime}$ given $s$ for $\tau+1$

$$
x_{s_{n} \mid s}^{(n)}(\tau+1)=\frac{x_{s_{n^{\prime}} \mid s}^{(n)}(t) e^{\beta^{(n)}\left(R_{s^{\prime} \mid s}^{(n)}(\tau+1)-R_{s_{n^{\prime}} \mid s}^{(n)}(\tau)\right)}}{\sum_{s_{n}^{\prime}}^{N} x_{s_{n^{\prime}} \mid s}^{(n)}(t) e^{\beta^{(n)}\left(R_{s^{\prime} \mid s}^{(n)}(\tau+1)-R_{s_{n}{ }^{\prime} \mid s}^{(n)}(\tau)\right)}},
$$

where $\beta^{(n)}$ is the learning rate for the unit $n$. We assume that the time scale of the update is larger than the relaxation time of the global Markovian process. The continuous time model by letting $\tau \Delta t \rightarrow t$ with $\tau \rightarrow \infty$ and $\Delta t \rightarrow 0$ is given as

$$
\frac{x_{s_{n^{\prime} \mid s}}^{(\dot{n})}(t)}{x_{s_{n^{\prime} \mid s}}^{(n)}(t)}=\beta^{(n)}\left(R_{s_{n^{\prime} \mid s}}^{(n)}(t)-R_{\mid s}^{(n)}(t)\right),
$$

for $n=1, \ldots, N$, where $R_{\mid s}^{(n)}=\sum_{s_{n^{\prime}}} x_{s_{n^{\prime}} \mid s}^{(n)} R_{s_{n^{\prime}} \mid s}^{(n)}$ is the conditional expectation of reinforcements over all possible symbols given the previous system state $s[1,7]$. Intuitively, when $\left(R_{s_{n^{\prime}} \mid s}^{(n)}(t)-R_{\mid s}^{(n)}(t)\right)$ is positive, that is, the conditional expectation reinforcement for a symbol $s_{n}{ }^{\prime}$ given $s$ is greater than the average of the expectation reinforcement given $s$, the logarithmic derivative of $x_{s_{n}{ }^{\prime} \mid s}^{(n)}(t)$ increases, and when negative, it decreases. The learning rate, $\beta^{(n)}$, controls the time scales of the adaptive dynamics of each unit $n$.

\section{Model dynamics}

Suppose that two biased coin tossing processes $X$ and $Y$ adaptively interact with each other. They produce a pair of symbols $i j$ at each time step, where $i$ and $j$ are either heads (0) or tails (1). At the next time step, $X$ sends a symbol $i^{\prime}$ to $Y$ based on the previous pair of symbols $i j$, and vice versa. If there is a causal interaction with one step memory, the global stochastic process becomes a simple Markovian process. When $X$ 's and $Y$ 's behaviors are causally separated, the whole system is a product of two biased coin tossing processes (case 10 in Fig. 1).

Considering Fig. 1, the extreme cases are 1 and 10. Case 1 corresponds to the situation, "each unit has one step memory of the previous global state $s$," and case 10 to, "no information of $s . "$ Local transition probabilities of $X$ and $Y$ are given as $\left(x_{i^{\prime} \mid i j}\right)=P\left(X^{\prime}=i^{\prime} \mid X=i, Y=j\right)$, and $\left(y_{j^{\prime} \mid i j}\right)=P\left(Y^{\prime}=j^{\prime} \mid X=i, Y=j\right)$, where $\sum_{i^{\prime}} x_{i^{\prime} \mid i j}=\sum_{j^{\prime}} y_{j^{\prime} \mid i j}=1$. The global Markovian kernel is given with $\left(x_{i^{\prime} \mid i j} y_{j^{\prime} \mid i j}\right)$ where $\sum_{i^{\prime}, j^{\prime}} x_{i^{\prime} \mid i j} y_{j^{\prime} \mid i j}=1$. When $X$ and $Y$ match heads (0) or tails (1) of coins (00 or 11), $Y$ reinforces the choice, and when they don't (01 or 10), $X$ reinforces the choice. 
(1)

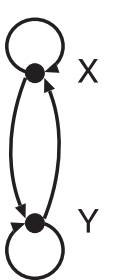

(6)

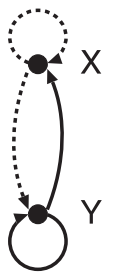

(2)

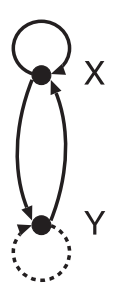

(7)

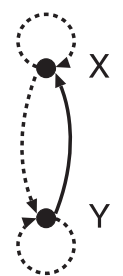

(3)

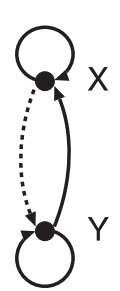

(8)

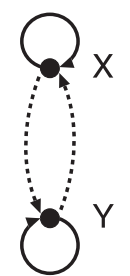

(4)

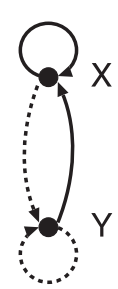

(9)

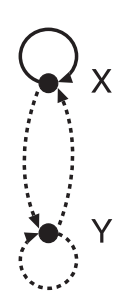

(5)

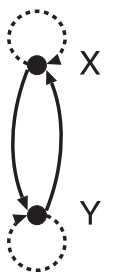

(10)

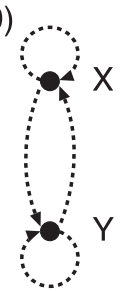

Fig. 1. Possible causal structure (case 1-10): $X \rightarrow Y$ indicates that $\mathrm{Y}$ receives symbols sent by $\mathrm{X}$ (information flow from $\mathrm{X}$ to $\mathrm{Y}$ ). Dashed arrows indicate ignorance of received symbols (no information flow).

This interaction is called the matching pennies game in game theory. The reinforcements are given by a bi-matrix

$$
(A, B)=\left(\left[\begin{array}{cc}
-\epsilon_{X} & \epsilon_{X} \\
\epsilon_{X} & -\epsilon_{X}
\end{array}\right], \quad\left[\begin{array}{cc}
\epsilon_{Y} & -\epsilon_{Y} \\
-\epsilon_{Y} & \epsilon_{Y}
\end{array}\right]\right),
$$

where $0<\epsilon_{X}, \epsilon_{Y}<1$. The interaction matrices, $A=\left(a_{i j}\right)$ and $B=\left(b_{j i}\right)$, are the reinforcements for $X$ and $Y$ for the global state $i j$. The Nash equilibrium of the game (4) in terms of game theory is an uniformly random state $(1 / 2,1 / 2)$. The conditional expectation reinforcements are given by $R_{i^{\prime} \mid i j}^{X}=$ $\left(A \mathbf{y}_{\mid i j}\right)_{i^{\prime}}$ and $R_{j^{\prime} \mid i j}^{Y}=\left(B \mathbf{x}_{\mid i j}\right)_{j^{\prime}}$, where $\mathbf{x}_{\mid i j}=\left(x_{0 \mid i j}, x_{1 \mid i j}\right)^{T}$, and $\mathbf{y}_{\mid i j}=\left(y_{0 \mid i j}, y_{1 \mid i j}\right)^{T}$. Equation (3) reduces to

$$
\begin{aligned}
& \frac{x_{i^{\prime} \mid i j}}{x_{i^{\prime} \mid i j}}=\beta^{X}\left[\left(A \mathbf{y}_{\mid i j}\right)_{i^{\prime}}-\mathbf{x}_{\mid i j} \cdot A \mathbf{y}_{\mid i j}\right], \\
& \frac{y_{j^{\prime} \mid i j}}{y_{j^{\prime} \mid i j}}=\beta^{Y}\left[\left(B \mathbf{x}_{\mid i j}\right)_{j^{\prime}}-\mathbf{y}_{\mid i j} \cdot B \mathbf{x}_{\mid i j}\right] .
\end{aligned}
$$

Equation (5) corresponds to adaptive dynamics for an interacting Markovian processes in an 8dimensional state space $\Pi_{i, j} \Delta_{i j}^{X} \times \Delta_{i j}^{Y}$, which is in the form of standard game dynamics.

Similarly, for case 10, we have

$$
\begin{aligned}
& \frac{x_{i^{\prime} \mid * *}^{\prime}}{x_{i^{\prime} \mid * *}}=\beta^{X}\left[\left(A \mathbf{y}_{\mid * *}\right)_{i^{\prime}}-\mathbf{x}_{\mid * *} \cdot A \mathbf{y}_{\mid * *}\right], \\
& \frac{y_{j^{\prime} \mid * *}^{\prime}}{y_{j^{\prime} \mid * *}}=\beta^{Y}\left[\left(B \mathbf{x}_{\mid * *}\right)_{j^{\prime}}-\mathbf{y}_{\mid * *} \cdot B \mathbf{x}_{\mid * *}\right],
\end{aligned}
$$

where $\mathbf{x}_{\mid * *}=\left(x_{0 \mid * *}, x_{1 \mid * *}\right)^{T}$, and $\mathbf{y}_{\mid * *}=\left(y_{0 \mid * *}, y_{1 \mid * *}\right)^{T}$. Here, the $*$ is either 0 or 1 , that corresponds to ignorance of received symbols. Equation (6) is, again, standard game dynamics in a 2-dimensional state space $\Delta^{X} \times \Delta^{Y}$. It is known that the dynamics of Eq. (6) is Hamiltonian with a constant of motion $H=1 / \beta^{X} D\left(\mathbf{x}^{*}|| \mathbf{x}\right)+1 / \beta^{Y} D\left(\mathbf{y}^{*}|| \mathbf{y}\right)$, where $D$ is Kullback-Leibler divergence, and where $\left(\mathbf{x}^{*}, \mathbf{y}^{*}\right)$ is the Nash equilibrium of the game $(A, B)$. The dynamics are neutrally stable periodic orbits for all range of parameters $\epsilon_{X}, \epsilon_{Y}[6,7]$. When the degree of freedom of the Hamiltonian systems is more than 2, the dynamics may be chaotic [11-13]. Summarizing, if all units have complete information of the previous global state $s$ (case 1), or they are all causally separated with no information of $s$ (case 10), the family of standard game dynamics recovers.

For intermediate cases 2-9, showing in Fig. 1, where units have partial information of $s$, we have explicit stationary distribution terms in the adaptive dynamics. Assuming through adaptation process, the transition probabilities are strictly positive, $0<x_{i^{\prime} \mid i j}, y_{j^{\prime} \mid i j}<1$, an unique stationary distribution $(p(i, j))$ exists. We denote the marginal stationary distributions $\mathbf{p}^{X}=(P(X=0), P(X=1))^{T}$, 
$\mathbf{p}^{Y}=(P(Y=0), P(Y=1))^{T}$. The conditional stationary distribution of $i$, given the previous state $j$, is denoted as $p(i \mid j)=p(i, j) / p(j)$, and those of $j$, given the previous state $i$, as $p(j \mid i)=p(i, j) / p(i)$. For case 2, with $R_{i^{\prime} \mid i j}^{X}=\left(A \mathbf{y}_{\mid i *}\right)_{i^{\prime}}$ and $R_{j^{\prime} \mid i *}^{Y}=\sum_{j} p(j \mid i)\left(B \mathbf{x}_{\mid i j}\right)_{j^{\prime}}$, Eq. (3) reduces to

$$
\begin{aligned}
& \frac{x_{i^{\prime} \mid i j}}{x_{i^{\prime} \mid i j}}=\beta^{X}\left[\left(A \mathbf{y}_{\mid i *}\right)_{i^{\prime}}-\mathbf{x}_{\mid i j} \cdot A \mathbf{y}_{\mid i *}\right], \\
& \frac{y_{j^{\prime} \mid i *}}{y_{j^{\prime} \mid i *}}=\beta^{Y}\left[\left(\sum_{j} p(j \mid i) B \mathbf{x}_{\mid i j}\right)_{j^{\prime}}-\mathbf{y}_{\mid i j} \cdot\left(\sum_{j} p(j \mid i) B \mathbf{x}_{\mid i j}\right)\right] .
\end{aligned}
$$

Similarly, for case 5, with $R_{i^{\prime} \mid * j}^{X}=\left(A \mathbf{p}^{X}\right)_{i^{\prime}}$ and $R_{j^{\prime} \mid i *}^{Y}=\left(B \mathbf{p}^{Y}\right)_{j^{\prime}}$, we obtain

$$
\begin{aligned}
& \frac{x_{i^{\prime} \mid * j}}{x_{i^{\prime} \mid * j}}=\beta^{X}\left[\left(A \mathbf{p}^{Y}\right)_{i^{\prime}}-\mathbf{x}_{\mid * j} \cdot A \mathbf{p}^{Y}\right], \\
& \frac{y_{j^{\prime} \mid i *}}{y_{j^{\prime} \mid i *}}=\beta^{Y}\left[\left(B \mathbf{p}^{X}\right)_{j^{\prime}}-\mathbf{y}_{\mid i *} \cdot B \mathbf{p}^{X}\right] .
\end{aligned}
$$

Note that $(p(i, j))$ are given as a function of $\left(x_{i^{\prime} \mid i j}\right)$ and $\left(y_{j^{\prime} \mid i j}\right)$, thus the equations of motion are in a closed form. For cases $2-9$, we have nonlinear couplings with a stationary distribution, which is in contrast to the quasi-linear coupling of standard game dynamics. Eqs. (6)-(8) are both in an extended form of standard game dynamics.

When the parameters are fixed to $\beta^{X}=\beta^{Y}$ and $\epsilon_{X}=\epsilon_{Y}=0.5$, we have four types of dynamics for all 10 cases in Fig. 1;

1. neutrally stable periodic motion of Markovian kernel,

2. convergence to a fixed Markovian kernel that gives a uniform stationary distribution,

3. sharp switching among almost deterministic Markovian kernel,

4. a combination of (1)-(3).

In contrast to the matching pennies game dynamics which shows only neutrally stable periodic orbits, it shows new types of dynamics naturally given by the Markovian structure. They are exemplified by case 1,2 , and 5 .

Case 1 (Eq. (5)): Neutrally stable quasi-periodic tori are observed. They are simply a product of periodic orbits in the matching pennies game dynamics. The dynamics of Eq. (6) is embedded in a subspace in the state space, given by $x_{i^{\prime} \mid 00}=x_{i^{\prime} \mid 01}=x_{i^{\prime} \mid 10}=x_{i^{\prime} \mid 11}$ and $y_{j^{\prime} \mid 00}=y_{j^{\prime} \mid 01}=y_{j^{\prime} \mid 10}=y_{j^{\prime} \mid 11}$ (Fig. 2, top).

Case 2 (Eq. (6)): A combination of the dynamics of Eq. (5), quasi-periodic tori, and the dynamics of Eq. (8), transients to a heteroclinic cycle, are observed (Fig. 2, middle). One of the infinitely many attracting periodic orbits, that corresponds to periodic orbits in Eq. (5), is selected depending on initial conditions (Fig. 2, middle).

Case 5 (Eq. (8)): Bi-stable dynamics is observed. A manifold which gives uniform stationary distribution $p(i, j)=1 / 4$, is an attracting set. Fixed points on this attracting manifold are all neutrally stable. Heteroclinic cycles which consists of several vertex saddles are also attracting sets. Depending on initial conditions, convergence to one of the fixed points on the attracting manifold or one of the heteroclinic cycles are observed (Fig. 2, bottom).

\section{Causal information flow}

In the standard game dynamics which describes a causally separated stochastic process, information flow is always 0. By using the Markovian extension of game dynamics, we can now quantify bidirectional information flow between stochastic units. 


\section{Case 1}
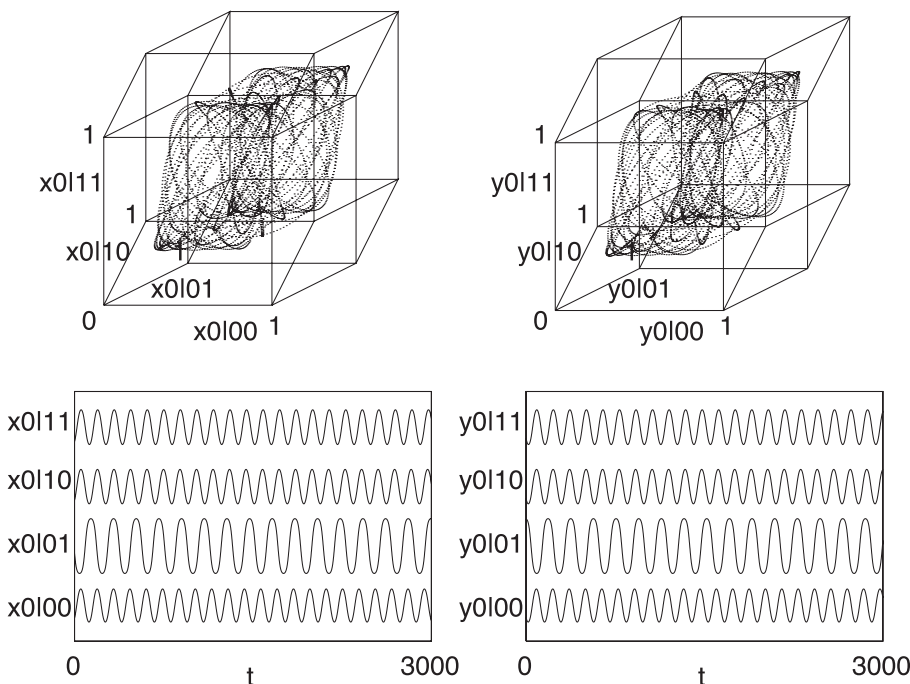

Case 2
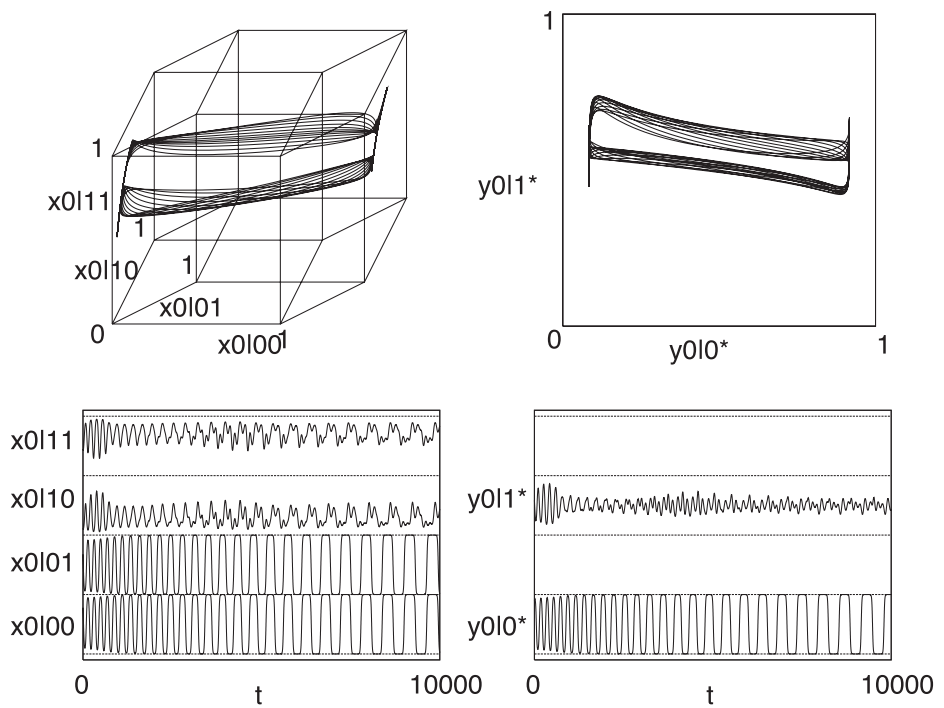

Case $5(a)$

Case 5 (b)
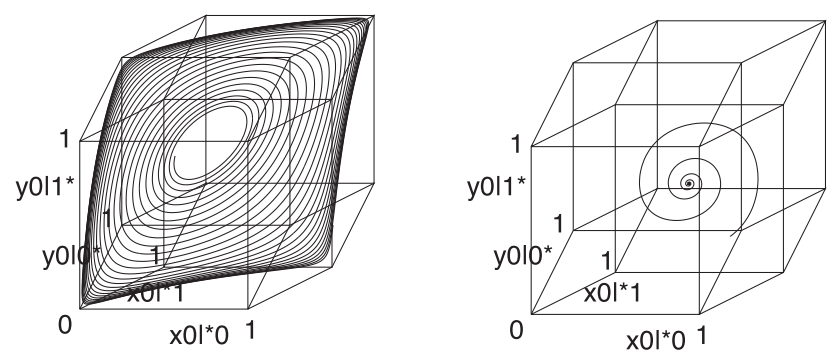

Fig. 2. (Top) Case 1: Neutrally stable quasi-periodic tori. (Middle) Case 2: A combination of quasi-periodic tori and transients to a heteroclinic cycle. (Bottom) Case 5: (a) Transients to a heteroclinic cycle which consists of vertex saddles $\left(x_{0 \mid * 0}, x_{0 \mid * 1}, y_{0 \mid 0 *}, y_{0 \mid 1 *}\right)=(0,0,0,0),(1,1,0,0),(0,0,1,1),(1,1,1,1)$ and (b) convergence to one of infinitely many neutrally stable fixed points which gives a uniform stationary distribution $p(i, j)=\frac{1}{4}$; (in this case, converging to $\left.\left(x_{0 \mid * 0}, x_{0 \mid * 1}, y_{0 \mid 0 *}, y_{0 \mid 1 *}\right)=(0.539057,0.460943,0.671772,0.328228)\right)$ are attracting sets. 
Equation (9) gives a measure of information flow which is conditional mutual information of $Y$ and $X^{\prime}$ given $X$. It is a measure of deviation of two random variables from stochastic independence of $X^{\prime}$ and $Y$, given $X$, and is sometime called transfer entropy $[8,9,14,15]$;

$$
\begin{aligned}
& I\left(Y: X^{\prime} \mid X\right) \\
= & \sum_{i^{\prime}, i, j} p\left(i^{\prime}, i, j\right) \log \frac{x_{i^{\prime} \mid i j}}{\sum_{j} p(j \mid i) x_{i^{\prime} \mid i j}} \\
= & -\sum_{i^{\prime}, i} p(i)\left(\sum_{j} p(j \mid i) x_{i^{\prime} \mid i j}\right) \log \left(\sum_{j} p(j \mid i) x_{i^{\prime} \mid i j}\right)+\sum_{i, j} p(i, j)\left[\sum_{i^{\prime}} x_{i^{\prime} \mid i j} \log \left(x_{i^{\prime} \mid i j}\right)\right] .
\end{aligned}
$$

Another measure of information flow which describes deviation of two random variables from causal independence, is formulated in [2], which is based on causality theory [10]. This causal information flow from $Y$ to $X^{\prime}$, given $X$, is defined by Eq. (10);

$$
\begin{aligned}
& I\left(Y \rightarrow X^{\prime} \mid X\right) \\
= & \sum_{i^{\prime}, i, j} p(i) p(j) x_{i^{\prime} \mid i j} \log \frac{x_{i^{\prime} \mid i j}}{\sum_{j} p(j) x_{i^{\prime} \mid i j}} \\
= & -\sum_{i^{\prime}, i} p(i)\left(\sum_{j} p(j) x_{i^{\prime} \mid i j}\right) \log \left(\sum_{j} p(j) x_{i^{\prime} \mid i j}\right)+\sum_{i, j} p(i) p(j)\left[\sum_{i^{\prime}} x_{i^{\prime} \mid i j} \log \left(x_{i^{\prime} \mid i j}\right)\right] .
\end{aligned}
$$

As a result, we have either stationary constant causal information flow or oscillatory causal information flow for all 10 cases in Fig. 1.

If $Y$ is a fixed information source, such as

$$
\left(y_{0 \mid 00}, y_{0 \mid 01}, y_{0 \mid 10}, y_{0 \mid 11}\right)=(1,0,1,0),
$$

the dynamics (5) with $\beta^{Y}=0$ monotonically converges to an optimal

$$
\left(x_{0 \mid 00}, x_{0 \mid 01}, x_{0 \mid 10}, x_{0 \mid 11}\right)=(0,1,0,1) .
$$

The system state $s$ is either 00 or 11 and $X$ is always rewarded. In this case, the transfer entropy defined by (9) eventually decreases to 0 ;

$$
\begin{aligned}
& I\left(X: Y^{\prime} \mid X\right)=0 \\
& I\left(Y: X^{\prime} \mid Y\right)=0 .
\end{aligned}
$$

In contrast, the causal information flow from $Y$ to $X$ shows a positive value;

$$
\begin{aligned}
I\left(X \rightarrow Y^{\prime} \mid X\right) & =0, \\
I\left(Y \rightarrow X^{\prime} \mid Y\right) & =\log 2 .
\end{aligned}
$$

As we interpret the model, there should be information flow from $Y$ to $X$ because $X$ receives symbols sent by $Y$ and extracts information from $Y$ 's behavior. Thus, $X$ is not stochastically dependent on $Y$, but it is causally dependent on $Y$. The above measure defined by (10) clearly captures this property. We can say that $I\left(X \rightarrow Y^{\prime} \mid X\right)$ is a more appropriate measure of the information flow.

In general game theoretic settings, causal information flow vanishes when the system state is on a manifold $M_{0}$ defined by $x_{i^{\prime} \mid i j}=\sum_{j} p(j) x_{i^{\prime} \mid i j}$ and $y_{j^{\prime} \mid i j}=\sum_{i} p(i) y_{j^{\prime} \mid i j}$, and is maximized to $\log 2$ when the system state is on a manifold $M_{1}$ defined by the set of points which have maximal distance from $M_{0}$. The case 5 , with this bi-stability between a fixed point and heteloclinic cycle, gives us an example of stationary causal information flow, which is similar to the above 'one-way' interaction example.

On the other hand, in the case 2 , we have oscillatory dynamics between the manifold $M_{0}$ and $M_{1}$, which emerges through reward-maximizing adaptation at each moment. In game theoretic situation, $X$ extracts information from $Y$ and $Y$ extracts information from $X$ as well. As a result, oscillatory behavior of information flow is observed (Fig. 4). This can be interpreted as the information flow in the dynamics switching among quasi-stationary states. 


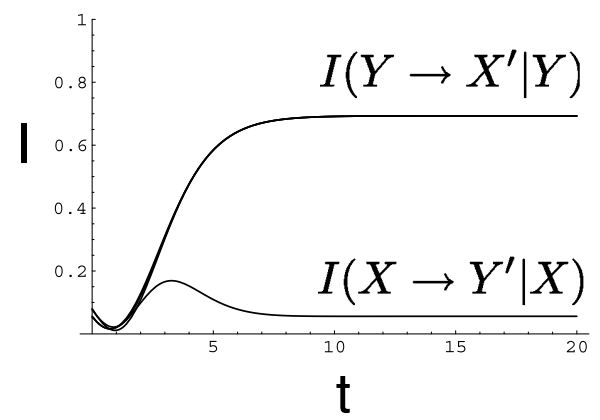

Fig. 3. $I\left(Y \rightarrow X^{\prime} \mid Y\right) \rightarrow \log 2$ which shows existence of causal information flow caused from the phenomenon that $X$ receives symbols sent by $Y$ and extracts information from $Y$. To the contrary, the transfer entropy, $I(X$ : $\left.Y^{\prime} \mid X\right)$, is always 0 .

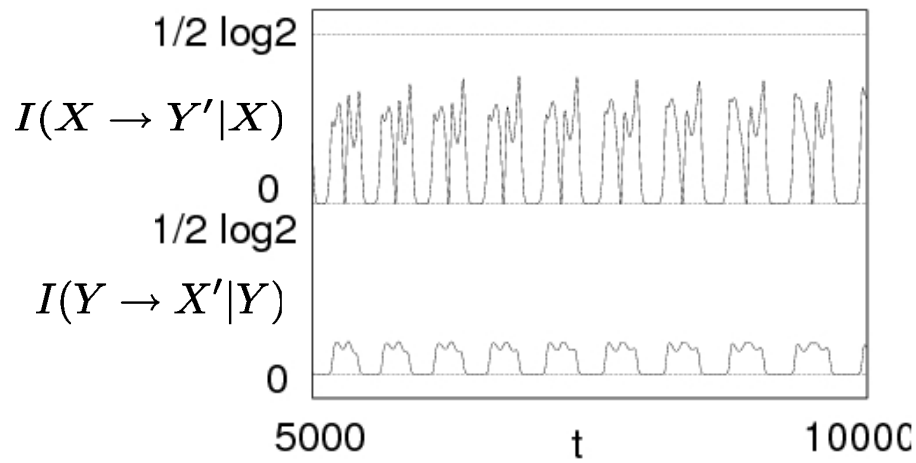

Fig. 4. (Top) Oscillatory behavior of causal information flow $I\left(X \rightarrow Y^{\prime} \mid X\right)$ (top) and $I\left(Y \rightarrow X^{\prime} \mid X\right)$ (bottom), in case 2. Oscillation of causal information flow emerges through reward-maximizing adaptation. These imply that the player X extracts information from the player $\mathrm{Y}$, and vice versa in the game theoretic situation.

\section{Conclusion}

The presented model is an extension of game dynamics to interacting Markovian processes. If all units have complete information of the previous global state $s$, or they are all causally separated without information of $s$, a family of standard game dynamics recovers. For intermediate cases with partial information of $s$, we have stationary distribution terms in the equations of motion. The extended model shows different behavior compared with the standard game dynamics $[5,6]$, which arise from underlying heterogeneous causal structure among players.

A measure of causal information flow [2] was applied to various of these structures, verifying its consistency with the prescribed causal connections. In particular, at an intuitive level, there should be no information flow from the player $X$ to the player $Y$, if there is no corresponding causal link. This intuition is perfectly captured by our measure. We also showed that, in more general situations, the causal information flow displays oscillatory behaviours, which, in particular, reflects the flow of information in both directions. In terms of dynamical systems theory, this oscillatory behaviour can be interpreted as switching among quasi-stationary states, which remains for future studies. Also, it is interesting to study adaptive dynamics for $N$ units with heterogeneous game interaction, and with various types of causal networks. The relationship individual reward structure and information flow among units may also give us new insights for game dynamics with asymmetric information structure.

\section{Acknowledgments}

Authors thank D. Albers (Columbia University, USA) for careful reading of the manuscript, D. Krakauer (Santa Fe Institute, USA) for useful discussions. YS is supported by JSPS Grant-in-Aid for Scientific Research (C) No. 26520201, (S) No. 26220044, and the external fellowship at London Mathematical Laboratory, UK. 


\section{References}

[1] N. Ay and I. Erb, "On a notion of linear replicator equations," Journal of Dynamics and Differential Equations, vol. 17, no. 2, pp. 427-451, 2005.

[2] N. Ay and D. Polani, "Information flows in causal networks," Adv. Compl. Syst., vol. 11, no. 1, pp. 17-41, 2008.

[3] V.S. Borkar, "Reinforcement learning in Markovian evolutionary games," Advances in Complex Systems, vol. 5, no. 1, pp. 55-72, 2002.

[4] T. Borgers and R. Sarin, "Learning through reinforcement and replicator dynamics," J. Econ. Theory, vol. 77, pp. 1-14, 1997.

[5] D. Fudenberg and D.K. Levine, "Theory of learning in games," MIT Press, 1998.

[6] J. Hofbauer and K. Sigmund, "Evolutionary games and population dynamics," Cambridge University Press: Cambridge, 1988.

[7] J. Hofbauer, "Evolutionary dynamics for bimatrix games: A hamiltonian system?," J. Math. Biol., vol. 34, pp. 675-688, 1996.

[8] K. Kaneko, "Lyapunov analysis and information flow in coupled map lattices," Physica, vol. 23 D, pp. 436-447, 1986.

[9] K. Matsumoto and I. Tsuda, "Noise induced order," Journal of Statistical Physics, vol. 31, pp. 87-106, 1983.

[10] J. Pearl, "Causality: models, reasoning, and inference," Cambridge Univ Press, 2000.

[11] Y. Sato, E. Akiyama, and J.D. Farmer, "Chaos in learning a simple two-person game," Proceedings of the National Academy of Sciences, vol. 99, pp. 4748-4751, 2002.

[12] Y. Sato and J.P. Crutchfield, "Coupled replicator equations for the dynamics of learning in multiagent systems," Phys. Rev., vol. E67, 015206(R), 2003.

[13] Y. Sato, E. Akiyama, and J.P. Crutchfield, "Stability and diversity in collective adaptation," Physica, vol. D 210, pp. 21-57, 2005.

[14] R. Shaw, "Dripping Faucet as a Model Chaotic System," Aerial Press, Santa Cruz, California, 1984.

[15] T. Schreiber, "Measuring information transfer," Phys. Rev. Lett., vol. 85, pp. 461-464, 2000.

[16] T. Wennekers and N. Ay, "Finite state automata resulting from temporal information maximization and a temporal learning rule," Neural Computation, vol. 17, pp. 2258-2290, 2005. 